EUROPEAN ORGANIZATION FOR NUCLEAR RESEARCH

European Laboratory for Particle Physics

Large Hadron Collider Project

LHC Project Report 445

\title{
MODIFIED BEAN MODEL AND FEM METHOD COMBINED FOR PERSISTENT CURRENT CALCULATION IN SUPERCONDUCTING COILS
}

\author{
C. Völlinger, M. Aleksa and S. Russenschuck
}

\begin{abstract}
Field variations in the LHC superconducting magnets, e. g. during the ramping of the magnets, induce magnetization currents in the superconducting material, the so-called persistent currents that do not decay but persist due to the lack of resistivity. This paper describes a semi-analytical hysteresis model for hard superconductors, which has been developed for the computation of the total field errors arising from persistent currents. Since the superconducting coil is surrounded by a ferromagnetic yoke structure, the persistent current model is combined with the finite element method (FEM), as the non-linear yoke can only be calculated numerically. The used finite element method is based on a reduced vector potential formulation that avoids the meshing of the coil while calculating the part of the field arising from the source currents by means of the Biot-Savart Law. The combination allows to determine persistent current induced field errors as function of the excitation and for arbitrarily shaped iron yokes. The model has been implemented into the ROXIE program and is tested using the LHC dipole magnet as an example.
\end{abstract}

LHC-ICP

Presented at the 9th International IGTE Symposium on Numerical Field Calculation in Electrical Engineering Graz, Austria, 11-14 September 2000

\footnotetext{
Administrative Secretariat

LHC Division

CERN

CH - 1211 Geneva 23

Switzerland

Geneva, 15 December 2000
} 


\title{
Modified Bean Model and FEM Method Combined for Persistent Current Calculation in Superconducting Coils
}

\author{
C. Völlinger, M. Aleksa, S. Russenschuck \\ CERN, 1211 Geneva 23, Switzerland
}

\begin{abstract}
index terms - persistent currents, magnetization, superconductor modeling
\end{abstract}

\section{Abstract}

Field variations in the LHC superconducting magnets, e. g. during the ramping of the magnets, induce magnetization currents in the superconducting material, the so-called persistent currents that do not decay but persist due to the lack of resistivity. This paper describes a semi-analytical hysteresis model for hard superconductors, which has been developed for the computation of the total field errors arising from persistent currents. Since the superconducting coil is surrounded by a ferromagnetic yoke structure, the persistent current model is combined with the finite element method (FEM), as the non-linear yoke can only be calculated numerically. The used finite element method is based on a reduced vector potential formulation that avoids the meshing of the coil while calculating the part of the field arising from the source currents by means of the Biot-Savart Law. The combination allows to determine persistent current induced field errors as function of the excitation and for arbitrarily shaped iron yokes. The model has been implemented into the ROXIE program and is tested using the LHC dipole magnet as an example.

\section{INTRODUCTION}

The Large Hadron Collider (LHC) [CERN,1995], a proton-proton superconducting accelerator, will consist of about 8400 superconducting magnet units of different types, all operating in superfluid helium at a temperature of $1.9 \mathrm{~K}$. The magnetic field components in the aperture of a magnet are expressed in the so-called multipoles which are the Fourier coefficients of the series expansion of the radial field component [Russenschuck,1998]. The multipoles are usually given in units of $10^{-4}$ relative to the main field component.

Since an extremely high field quality is needed for the storage of an intense particle beam for many hours, the relative deviation from the ideal field in the aperture of a magnet should not exceed a few parts in units of $10^{-4}$. In superconducting magnets, the field quality in the aperture is determined by the coil arrangement and the position of the conductors. These conductors are slightly keystoned, i. e. they have trapezoidal shape, in such a way as to give a good approximation of the ideal (cosine theta) distribution [CERN,1996]. Even small deviations in current positions will cause a considerable loss of field quality in the aperture. The implications for the numerical field calculation of these magnets are:

- The coil has to be modelled very precisely, as the current density of the conductors is non-uniform due to the different compaction of strands on the cable's narrow and wide side.

It is therefore advantageous to use the finite element method based on a reduced vector potential formulation in order to avoid the meshing of the coil.

- The influence of the so-called persistent currents that disturb the ideal current distribution in the coil, especially at low field level, has to be modelled using a hysteresis model for hard superconductors. The density of the magnetization due to these persistent currents also varies in the conductor due to different filling factors on the cable's narrow and wide side.

The use of a reduced vector potential formulation [Bíró,1998] that avoids the meshing of the coil completely also limits the numerical errors to the part contributed by iron magnetization. This principle has been incorporated into the ROXIE program [Russenschuck,1999] at CERN in the framework of a collaboration with the University of Graz and is now combined with a semi-analytical model for persistent current computation.

\section{The Superconducting Filament Model}

The coils of the LHC main dipole magnet are wound of a keystoned Rutherford type cable, containing 28 and 36 wires on the inner and the outer coil layer, respectively. The NbTi-strands of the cable are made of filaments of 6 and $7 \mu \mathrm{m}$ in diameter (inner and outer layer of the coil). According to the Bean-model [Bean,1964], a hard superconductor tries to screen the external field changes by generating a screening current distribution of critical density $J_{c}$. In order to simulate the magnetic field produced by single filaments in an external field, they are discretized in elliptical layers as shown in Fig. 1. The Bean 
model has been modified insofar, as the current distribution in each elliptical layer depends on the actual field level experienced. Each layer produces a dipole screening field reducing the external field inside the superconductor. Since the inner layers of the discretized filament are shielded by the screening currents prescribed on the outer layer, they can transport a higher current density. M. Wilson [Wilson,1983] has shown, that current distribu-

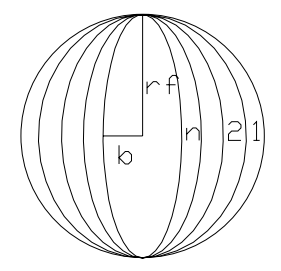

Fig. 1. Discretized filament with elliptically layered current distribution

tions in filaments can be modelled as elliptically shaped, if the magnetic field outside the filament is of interest and the applied field in the cross-section is perpendicular to the filament axis but not necessarily fully penetrating.

The screening field and the magnetization of one elliptical current layer is given by eqs. (1) in case of circular outer shape. In case of non-circular outer shape, the current layers have to be superposed. Here the magnetization caused by the induced screening currents is defined as the magnetic moment per unit volume.

$$
\begin{aligned}
\mathrm{B}_{\text {screen }} & =\frac{2 \mu_{0} \mathrm{~J}_{\mathrm{c}} \mathrm{r}_{\mathrm{f}}}{\pi}\left(1-\sqrt{1-\varepsilon^{2}} \frac{\arcsin (\varepsilon)}{\varepsilon}\right) \text { in }[T] \\
\mathrm{M} & =\frac{4}{3 \pi} \mathrm{r}_{\mathrm{f}} \mathrm{J}_{\mathrm{c}} \varepsilon^{2} \text { in }[A / m]
\end{aligned}
$$

The parameter $\varepsilon$ represents the ellipticity and $\mathrm{r}_{\mathrm{f}}$ the filament radius. In case the local external field exceeds the maximum screenable value of the filament (fully penetrated state), the magnetization takes its peak value where $\varepsilon$ equals 1 .

Each layer of the discretized filament can flip individually in case the external field changes orientation. This allows to account for local field variations in the coil arising from yoke saturations during the ramping of the magnets.

The critical current density $J_{\mathrm{c}}$ is now considered as an intrinsic material property, whose dependence on the magnetic induction is taken into account by a current fit function [Bottura,1999]:

$$
\begin{aligned}
& J_{\mathrm{c}}=f(B, T)=\frac{C_{0}}{B}\left(\frac{B}{B_{\mathrm{c} 2}}\right)^{\alpha}\left(1-\frac{B}{B_{\mathrm{c} 2}}\right)^{\beta}\left(1-\left(\frac{T}{T_{\mathrm{c} 0}}\right)^{n}\right)^{\gamma} \\
& \begin{array}{rlllll}
\text { where } & & & & & \\
B_{\mathrm{c} 2}= & 14.5 \mathrm{~T} & \text { upper critical field } & \alpha= & 0.57 & \text { fit param. } \\
T_{\mathrm{c} 0}= & 9.2 \mathrm{~K} & \text { critical temperature } & \beta= & 0.9 & \text { fit param. } \\
C_{0}= & 27.04 & \text { fit parameter } & \gamma= & 2.32 & \text { fit param. } \\
n= & 1.7 & \begin{array}{l}
\text { number from } \\
\text { [Lubell,1983] }
\end{array} & & &
\end{array}
\end{aligned}
$$

The current density of one elliptically shaped layer can be considered as constant in case the layers are sufficiently thin. $B_{\mathrm{p}}$, the maximum field that can be screened by a filament, does not have to be measured. It can be computed beforehand by considering the centre of the filament as completely screened and calculating reversely to the maximum screenable field on the filament surface, taking into account the dependence on the current density.

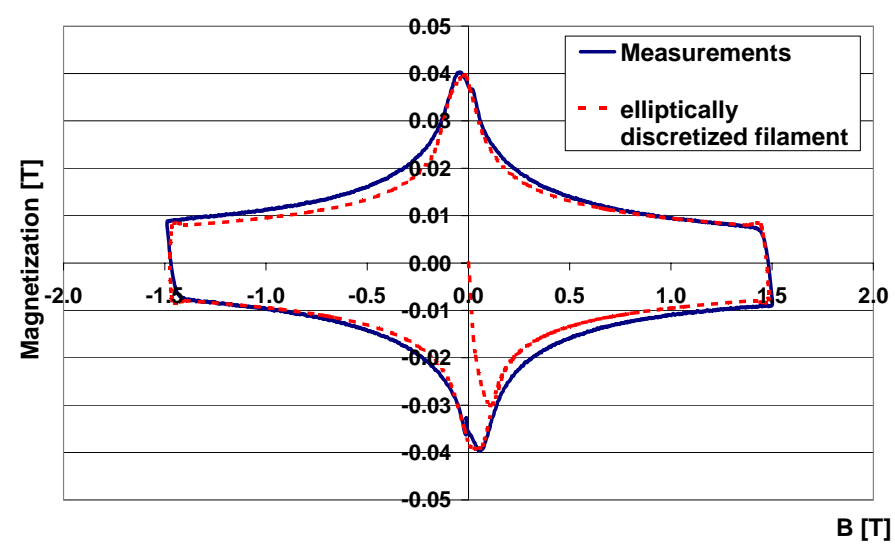

Fig. 2. Computed magnetization curve (dashed line) of a superconducting NbTi-filament compared with measurements [Le Naour,1998] (continues line).

A complete up-down-ramp cycle (here $0 \mathrm{~T} \rightarrow 1.5 \mathrm{~T}$ $\rightarrow-1.5 \mathrm{~T} \rightarrow 1.0 \mathrm{~T}$ ) for a filament compared with measurements [Le Naour,1998] is shown in Figure 2 in order to demonstrate the hysteretic behaviour. The curve also shows that the shift of the magnetization curve with respect to the ordinate axis (hysteretic effect due to the persistence of induced currents while passing the null-value of the external field) arises without being explicitly modeled.

Figure 3 shows a flow chart of the algorithm for the computation of persistent currents, implemented in the ROXIE 8.0 program. As can be seen, the magnetization currents are computed and added to the source currents. Re-iterations with updated source fields are performed until convergence is obtained. The parameters shown in the flow chart for the computation of persistent currents are as follows: $\mathrm{I}_{\mathrm{S}}$ presents the total current (source current) driven during one LHC cycle while $I_{n}$ is the individual transport current in single strands. $\mathbf{H}_{\mathbf{S}, \mathbf{i}}$ is the source field at the i-th strand position and is calculated from the BiotSavart Law. $A_{R}$ is the $\mathrm{z}$-component of the reduced vector potential arising from the surrounding ferromagnetic non-linear (iron) yoke. The iron magnetization $\mathbf{M}^{\mathbf{I R O N}}$ is defined as the magnetic moment per unit volume. $\mathbf{H}_{\mathbf{R}, \mathbf{i}}^{\text {IRoN }}$ represents the reduced magnetic induction due to the iron magnetization. Hence, one gets the magnetic field at the i-th strand position $\mathbf{H}_{\mathbf{i}}$ by superposing the source field and the reduced field.

$\mathbf{M}_{\mathbf{i}}^{\text {PERs }}$ represents the superconducting filament magnetization, which is computed with a semi-analytical hysteresis model for hard superconductors. Iterations in form of re-calculations with updated source fields are performed until convergence shows. The parameter $\mathbf{B}_{\mathbf{R}, \mathbf{i}}^{\mathbf{P E R}}$ denotes 

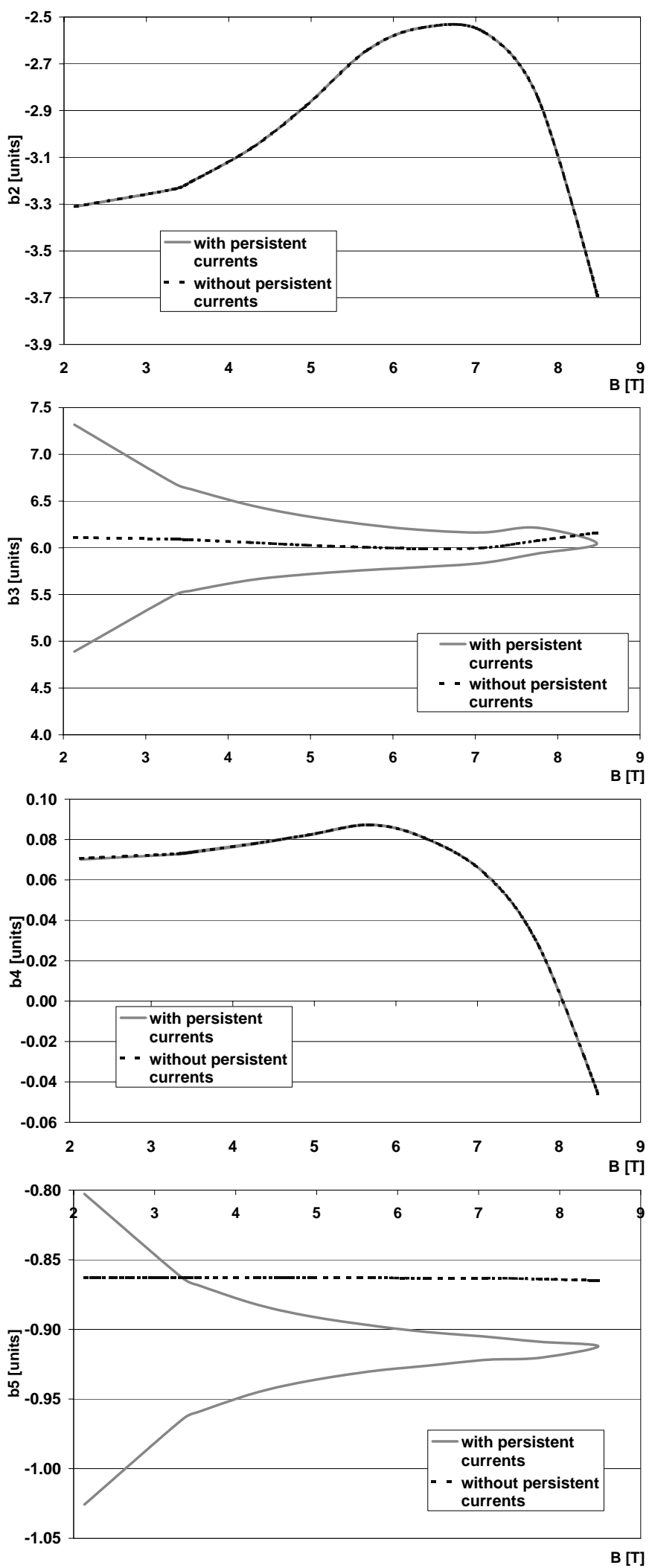

Fig. 5. Multipoles $\mathrm{b}_{n}$ in units of $10^{-4} @ 17 \mathrm{~mm}$ reference radius during current ramping (LHC up- and downramp-cycle); dashed lines: without persistent currents, continuous lines: taking persistent currents into account
Expected multipoles $b_{\mathrm{n}}$ in units of $10^{-4} @ 17 \mathrm{~mm}$ reference radius during current ramping (LHC upramp cycle), taking into account the persistent

\begin{tabular}{|c|c|c|c|}
\hline & $\begin{array}{c}b_{n} \text { at } \\
\text { injection field }\end{array}$ & $\begin{array}{c}b_{n} \text { at } \\
\text { nominal field }\end{array}$ & $\begin{array}{c}\Delta b_{n} \\
\text { (up-ramp) }\end{array}$ \\
\hline $\mathrm{b}_{2}$ & -3.064 & -3.442 & 1.146 \\
\hline $\mathrm{b}_{3}$ & -1.376 & 6.787 & 9.435 \\
\hline $\mathrm{b}_{4}$ & 0.080 & -0.032 & 0.133 \\
\hline $\mathrm{b}_{5}$ & -0.132 & -0.912 & 0.894 \\
\hline $\mathrm{b}_{6}$ & 0.004 & -0.002 & 0.006 \\
\hline $\mathrm{b}_{7}$ & 0.242 & 0.631 & 0.445 \\
\hline
\end{tabular}

\section{Conclusion}

A hysteresis model for hard superconducting filaments has been developed in order to compute persistent current induced multipole errors in the superconducting coils of LHC magnets. The model considers

- non-fully penetrated filaments in the coil crosssection

- dependence of current density distribution along the filament radius

- the hysteretic behaviour of hard superconducting material

- changes in external field orientation

Simulations of up- and downramp cycles in the LHC dipoles considering the persistent currents show a strongly hysteretic behaviour of the odd multipoles and a slightly hysteretic behaviour of the even multipoles.

\section{ACKnowledgements}

The authors would like to thank R. Wolf and S. Le Naour from LHC-MMS group at CERN for putting the measuring results at our disposal.

\section{REFERENCES}

[CERN,1995] The LHC study group. The Yellow Book, LHC, The Large Hadron Collider - Conceptual Design. CERN/AC/955(LHC), Geneva, Switzerland, 1995.

[Russenschuck,1998] Stephan Russenschuck. Field Quality in Accelerator Magnets. In Proceedings to the 1st International ROXIE Users Meeting and Workshop, Geneva, Switzerland, 1998.

[CERN,1996] S. Turner (ed.). Superconductivity in Particle Accelerators. CERN/CAS96-03, Geneva, Switzerland, 1996.

[Bíró,1998] Oszkár Bíró. The Use of a Reduced Vector Potential Formulation for the Calculation of Iron Induced Field Errors. In Proceedings to the 1st International ROXIE Users Meeting and Workshop, Geneva, Switzerland, 1998.

[Russenschuck,1999] Stephan Russenschuck. ROXIE: Routine for the Optimization of Magnet X-Sections, Inverse Field Calculation and Coil End Design. CERN, Geneva, 1999.

[Bean,1964] Charles P. Bean. Magnetization of High Field Superconductors. In Review of Modern Physics, vol. 36, p. 31-39, 1964 . 
[Wilson,1983] Martin N. Wilson. Superconducting Magnets. Monographs on Cryogenics, Oxford University Press, New York, 1983.

[Bottura,1999] Luca Bottura. A Practical Fit for the Critical Surface of NbTi. In 16th International Conference on Magnet Technology, Florida, USA, 1999.

[Lubell,1983] M. S. Lubell. Empirical Scaling Formulas for Critical Current and Critical Field for Commercial NbTi. In IEEE Transactions on Magnetics, vol. 19, no. 3, 1983.

[Le Naour,1998] S. Le Naour, L. Oberli, R. Wolf, R. Puzniak, A. Szewczyk, A. Wisniewski, H. Fikis, M. Foitl, and H. Kirchmayr. Magnetization Measurements on LHC Superconducting Strands. In Applied Superconductivity Conference, Palm Springs, USA, 1998. 